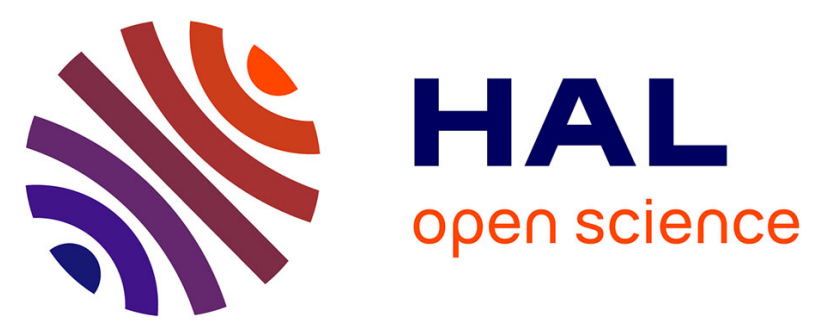

\title{
Rarefied Pure Gas Transport in Non-Isothermal Porous Media: Effective Transport properties from Homogenization of the Kinetic Equation
}

\author{
Gérard Vignoles, Pierre Charrier, Christophe Preux, Bruno Dubroca
}

\section{- To cite this version:}

Gérard Vignoles, Pierre Charrier, Christophe Preux, Bruno Dubroca. Rarefied Pure Gas Transport in Non-Isothermal Porous Media: Effective Transport properties from Homogenization of the Kinetic Equation. Transport in Porous Media, 2007, 73, pp.211-232. 10.1007/S11242-007-9167-7 . hal00269369

\section{HAL Id: hal-00269369 \\ https://hal.science/hal-00269369}

Submitted on 15 Jan 2009

HAL is a multi-disciplinary open access archive for the deposit and dissemination of scientific research documents, whether they are published or not. The documents may come from teaching and research institutions in France or abroad, or from public or private research centers.
L'archive ouverte pluridisciplinaire HAL, est destinée au dépôt et à la diffusion de documents scientifiques de niveau recherche, publiés ou non, émanant des établissements d'enseignement et de recherche français ou étrangers, des laboratoires publics ou privés. 


\title{
RAREFIED PURE GAS TRANSPORT IN NON-ISOTHERMAL POROUS MEDIA : EFFECTIVE TRANSPORT PROPERTIES FROM HOMOGENIZATION OF THE KINETIC EQUATION
}

Gérard L. Vignoles ${ }^{1}$ (vinhola@lcts.u-bordeaux1.fr)

1. Université Bordeaux l,

Lab. for ThermoStructural Composites (LCTS),

UMR 5801 CNRS-CEA-Safran-UBl,

3, Allée La Boëtie,

F-33600 Pessac, France

Pierre Charrier $^{2}$ (pierre.charrier@math.u-bordeaux1.fr)

, Christophe Preux ${ }^{2,3}$ (christophe.preux@math.u-bordeaux1.fr)

and Bruno Dubroca ${ }^{2,3}$ (bruno.dubroca@math.u-bordeaux1.fr)

2. Université Bordeaux l,

Applied Mathematics of Bordeaux (MAB),

UMR 5466 CNRS-UB1, LRC-CEA M03,

351 Cours de la Libération,

F-33405 Talence Cedex, France

3. Commissariat à l'Energie Atomique (CEA)

Centre d'Etudes Scientifiques et Techniques d'Aquitaine, BP2, F-33114 Le Barp, France

\begin{abstract}
Viscous flow, effusion, and thermal transpiration are the main gas transport modalities for a rarefied gas in a macro-porous medium. They have been well quantified only in the case of simple geometries. This paper develops a model based on the homogenization of kinetic equations producing effective transport properties (permeability, Knudsen diffusivity, thermal transpiration ratio) in any porous medium sample, as described $e . g$. by a digitized 3D image. The homogenization procedure neglecting the effect of gas density gradients on heat transfer through the solid - leads to macroscopic transfer relations, and to closure problems in $\mathbb{R}^{6}$ for the obtention of effective properties. Coherence of the approach with previous literature on the subject is discussed. The asymptotic limits of the model (rarefied and continuum regimes) are also studied. One of the main results is that the effect of the geometry on thermal transpiration has to be described by a tensor which is distinct from the permeability and Knudsen diffusion tensors.
\end{abstract}

Keywords: Knudsen diffusion, thermal transpiration, kinetic equation, homogenization 


\section{List of symbols}

\section{Latin}

- $a$ : Velocity of sound $\left(m . s^{-1}\right)$

- a : Thermal diffusivity $\left(m^{2} \cdot s^{-1}\right)$

- $A_{g}$ : Parameter in eq. (15) (-)

- $b(\mathbf{z}, \omega)$ : Scattering kernel $\left(m^{3}\right)$

- $B$ : Porous medium intrinsic permeability $\left(m^{2}\right)$

- c : Microscopic molecular velocity

- $\bar{c}$ : Mean quadratic velocity $\left(m . s^{-1}\right)$

- $C$ : Gas concentration $\left(\mathrm{mol} \cdot \mathrm{m}^{-3}\right)$

- $C^{\prime}$ : Total mole number including dust molecules per unit volume $\left(\mathrm{mol} . \mathrm{m}^{-3}\right)$

- $C_{d}$ : Dust mole number per unit volume $\left(\mathrm{mol} . \mathrm{m}^{-3}\right)$

- $C_{v}$ : Specific heat capacity at constant volume $\left(J . \mathrm{kg}^{-1} \cdot \mathrm{K}^{-1}\right)$

- $\partial Y_{f s}$ : Interface between domains $Y_{f}$ and $Y_{s}$

- $\underline{\underline{D}}$ : Effective tensor linking mass flux and density gradient $\left(m^{2} \cdot s^{-1}\right)$

- $\underline{\underline{D}}$ : Effective tensor linking mass flux and temperature gradient $\left.\overline{\overline{(k}} g \cdot m^{-1} \cdot s^{-1} \cdot K^{-1}\right)$

- $D_{g d}$ : Gas-dust diffusion coefficient $\left(m^{2} \cdot s^{-1}\right)$.

- $D_{K}:$ Knudsen diffusion coefficient $\left(\mathrm{m}^{2} \cdot \mathrm{s}^{-1}\right)$.

$-\quad d_{p}$ : Pore diameter $(m)$

- E : Internal (translational) energy $\left(J . \mathrm{kg}^{-1}\right)$

- $\underline{\underline{\mathcal{E}}}$ : Translational energy tensor $\left(J . \mathrm{kg}^{-1}\right)$

- $\mathbf{F}$ : External applied force $\left(N \cdot \mathrm{mol}^{-1}\right)$

- $f$ : Mass distribution function in space and velocity spaces $\left.\left(k g \cdot m^{-9} \cdot s^{3}\right)\right)$ 
- J : Molar flux (mol. $\left.m^{2} \cdot s^{-1}\right)$.

- $\underline{\underline{K}}$ : Effective heat conductivity tensor $\left(W \cdot m^{-1} \cdot K^{-1}\right)$

- $k_{B}$ : Boltzmann's constant $\left(J . K^{-1}\right)$

- $K e_{2}$ : Viscous to effusion flux ratio (-)

- Kn: Knudsen number (-)

- $\bar{l}$ : Mean free path $(m)$

- L : Linearized collision operator $\left(\mathrm{kg} \cdot \mathrm{m}^{-9} \cdot \mathrm{s}^{-4}\right)$

- $L_{*}:$ Reference length $(m)$

- $M$ : Molecular mass $\left(k g \cdot \mathrm{mol}^{-1}\right)$

- $M(f)$ : Maxwellian velocity distribution $\left(m^{-4} \cdot s^{4}\right)$

- $M_{n}(f)$ : Maxwellian distribution normalized to unity for half-flux $\left(m^{-4} \cdot s^{4}\right)$

- Ma: Mach number (-)

- $n$ : Molecule number per unit volume (molecules. $m^{-3}$ )

- $n^{\prime}$ : Total molecule number including dust molecules per unit volume (molecules. $m^{-3}$ )

- $n_{d}$ : Dust molecule number per unit volume (molecules. $m^{-3}$ )

- $\mathbf{n}$ : Normal vector (-)

- $P$ : Pressure $(P a)$

- Pr: Prandtl number (-)

- $Q(f, f)$ : Collision operator $\left(k g \cdot \mathrm{m}^{-9} \cdot \mathrm{s}^{2}\right)$

- $r$ : Dust particle radius $(m)$

- $\mathcal{R}=\mathcal{N}_{A} k_{B}:$ Perfect gas constant $\left(J \cdot \mathrm{mol}^{-1} K^{-1}\right)$

- $R_{0}$ : Geometrical factor in eq. $(7)(m)$

- Re : Reynolds number (-) 
- $S_{d}:$ Gas-dust collision section $\left(m^{2}\right)$

- $T:$ Temperature $(K)$

- $T_{c}:$ Critical temperature $(K)$

- $\underline{\underline{T}}$ : Tensor entering the definition of the ES-BGK approximation of the scattering operator $\left(J \mathrm{~kg}^{-1}\right)$

- $\mathbf{u}$ : Intrinsic mass-average gas velocity $\left(m . s^{-1}\right)$

- $\mathbf{v}$ : Extrinsic mass-average gas velocity $\left(m . s^{-1}\right)$

- $\mathbf{w}$ : Mute integration variable for molecular velocity $\left(\mathrm{m} . \mathrm{s}^{-1}\right)$

- $\mathbf{x}$ : Space coordinates $(m)$

- $\mathbf{x}^{\prime}$ : Large-scale space coordinate $(m)$

- $\mathbf{y}$ : Small-scale space coordinate $(m)$

- $Y$ : Small space subset

\section{Greek}

- $\alpha$ : Closure variable $\left(m . K^{-1}\right)$

- $\left(\alpha_{g d}\right)_{t r}$ : Gas-dust generalized thermal diffusion ratio, for which the only translational contribution of molecules has been retained $(-)$

- $\alpha_{T}$ : Thermal diffusion ratio (-)

- $\beta$ : Closure variable $\left(m^{4} \cdot k^{-1}\right)$

- $\gamma$ : Closure variable $(m)$

$-\gamma$ : Compressibility factor (-)

$-\Delta$ : Second-order correction to diffusion coefficients (-)

$-\epsilon$ : Porosity (-)

- $\varepsilon:$ Small parameter for asymptotics (-)

- $\eta$ : Gas viscosity (Pa.s)

- $\zeta$ : Intersection point of a molecular trajectory with a wall $(m)$ 
- $\underline{\underline{\Theta}}$ : Tensor entering the definition of the ES-BGK approximation of the scattering operator $\left(J . \mathrm{kg}^{-1}\right)$

- $\lambda$ : Thermal conductivity $\left(W \cdot m^{-1} \cdot K^{-1}\right)$

- $\left(\lambda_{i i}\right)_{t r}$ : Translational component of thermal conductivity $\left(W \cdot m^{-1} \cdot K^{-1}\right)$

- $\nu$ : Kinematic viscosity $\left(m^{2} \cdot s^{-1}\right)$

- $\quad \nu$ : Adjustable coefficient for the ES-BGK model (-)

- $\xi$ : Correction factor accounting for non-specular collisions (-)

- $\pi_{g}, \pi_{g}^{\prime}$ : Pressure correction parameters for Knudsen diffusion $(P a)$

- $\rho:$ Gas density $\left(k g \cdot m^{-3}\right)$

- $\rho_{s}:$ Solid density $\left(\mathrm{kg} \cdot \mathrm{m}^{-3}\right)$

- $\sigma:$ Accommodation factor (-)

- $\sigma_{c}$ : Collision diameter $(m)$

- $\sigma_{v}$ : Internal surface area $\left(m^{-1}\right)$

- $\Sigma:$ Fluid/solid interface

$-\Sigma_{c}$ : Collision cross-section $\left(m^{2}\right)$

- $\tau$ : Relaxation time $(s)$

- $\underline{\underline{\tau}}$ : Tortuosity (-)

- $\psi$ : Any function in $\mathbb{R}_{c}^{3}$ velocity space (-)

- $\omega$ : Orientation $(s r)$

- $\Omega$ : Open set of $\mathbb{R}^{3}$ position space

- $\Omega_{v}:$ Collision integral for gas viscosity (-)

- $\Omega_{g d}$ : Collision integral for gas-dust diffusion (-)

- $\otimes:$ Tensor product or outer product

\section{Subscripts and underscripts}

$-\cdot$ vector in $1, \mathbf{c},|c|^{2}$ space 
- $[\cdot]_{1}$ : first-order approximation in DGM derivation

$-{ }_{c}$ : relative to gas critical point

$-\cdot_{d}$ : relative to dust

- $\cdot_{f}$ : relative to fluid phase

$-{ }_{g}$ : relative to gas species

- ${ } K$ : relative to Knudsen transport

- $\cdot_{p}$ : relative to pore $(\mathrm{s})$

$-\cdot_{s}$ : relative to solid phase

- $\cdot_{t r}$ : translational contribution

${ }_{-}{ }_{v}$ : relative to viscous transport

${ }_{-} \cdot_{*}$ : reference quantity

\section{Introduction}

This work has been motivated by several physico-chemical studies, among which the study of CVI (Chemical Vapor Infiltration) (Naslain and Langlais, 1990) and its variants, especially including a thermal gradient, like TG-CVI (Golecki, 1997; Leutard et al., 2002), the rapiddensification process (Narcy et al., 1995; Bruneton et al., 1997), and the forced-CVI process (Lackey, 1989; Vaidyaraman et al., 1995). Such processes are privileged routes for the fabrication of high-quality carbon-carbon and other ceramic-ceramic composites (Naslain, 1999), which are used in various aerospace applications, as well as in braking technology. They involve the penetration of a hot fibrous medium by reactant gases which are transported and eventually react, either homogeneously (by pyrolysis), or heterogeneously, thus yielding a ceramic or carbon deposit, which is going to act as the matrix of the fiber-reinforced composite. Since high temperatures and low pressures are required, and that the pore sizes may be as low as a fraction of a micrometer, the description of the gas transport in such a process is rather involved. Indeed, it features a combination of viscous flow, interspecies diffusion and Knudsen flow, also called "free molecular 
flow", "rarefied gas flow", "effusion", "Klinkenberg regime", etc .... The intermediate regime between viscous flow and effusion is often referred to as "slip-flow regime", or "Darcy-Klinkenberg regime".

It is also to be pointed that when ceramic-ceramic composites are in their nominal conditions of use, they suffer chemical damage from the surrounding atmosphere ; the mechanisms of such a degradation also involve gas transport and heterogeneous reaction at high temperatures and low pore dimensions (Lamouroux and Camus, 1994).

Except in isothermal CVI condition, thermal gradients are present in the studied porous media, and their role may be large, since chemical reactions are usually highly dependent on temperature. Gas transport is also affected by thermal gradients through various couplings, among which $: i$ ) the perfect gas law which states that density is inversely proportional to temperature, and $i i$ ) the dependance of the transport coefficients (viscosity, binary and Knudsen regime diffusivities) on temperature (Hirschfelder et al., 1963). The additional phenomena that also has to be accounted for is the possibility of mass transport due to thermal gradients, which is predicted by the classical Linear Irreversible Thermodynamic theory (Hirschfelder et al., 1963), and has two expressions inside porous media : $i$ ) the intermolecular Soret effect (thermodiffusion), which is gas segregation under the action of temperature gradients, and $i$ ) thermal transpiration (Mason and Malinauskas, 1983), which is the equivalent of Knudsen diffusion with respect to thermodiffusion.

Rarefied gas flow in isothermal and non-isothermal conditions have been described in past works, leading to the phenomenological frames known as "Dusty-Gas Model" (Mason and Malinauskas, 1983) and "Binary Friction Model" (Kerkhof, 1996). They provide averaged transport equations for porous media considered as being homogeneous. The "Dusty-Gas Model" (DGM) approach is based on the consideration of Stefan-Maxwell diffusion laws in the limit that one of the gaseous components (the "dust") becomes large in size and weight, and is held fixed with respect to the laboratory frame. This model makes use of kinetic theory results, and uses the fact that up to firstorder approximation, the viscous and diffusive fluxes are independent from each other. However, a thorough discussion by Kerkhof (Kerkhof, 1996) points out that equations based on gas-gas momentum transfer are used in the case where no such transfer predominates or even occurs at all. As an alternative to the DGM, the author proposes the 
"Binary Friction Model" (BFM), which insists on the combination in parallel of Knudsen and viscous transport, both in series with binary diffusion, and represents the effective forces exerted on the fluid components as resulting from frictional contributions arising from the various transport modalities. This model starts from the already averaged equations for the transport modalities, with poreaveraged macroscopic quantities, and combines the contributions in an appropriate manner. However, it is to be pointed out that it could be physically incorrect to use geometrical averaging before using the moments approximations in the rarefied regime. This point needed to be checked out, and this is one of the questions addressed in this paper.

The identification of transport coefficients, and in particular of the precise impact of the pore geometries, has been treated mostly in isothermal conditions. The limiting cases of pure viscous flow (i.e. Darcy regime), pure diffusive flow, and pure Knudsen flow have been often separately addressed. Other works have considered the transition between two of the three transport modalities, mainly binary and Knudsen diffusion, by means of random walk algorithms in idealized 3D images of porous media (Burganos and Sotirchos, 1989; Tomadakis and Sotirchos, 1991) or by variational methods applied to media considered through their $n$-point correlation distributions (Strieder and Prager, 1968; Strieder, 1971). The consideration of slip-flow in addition to viscous flow in a change-of-scale methodology has been formally presented in (Whitaker, 1987). Homogenization performed on the same problem has been dealt with in (Skjetne and Auriault, 1999).

Thermal transpiration has been studied by numerous authors on the basis of kinetic equations, but almost always in the geometry of a straight cylindrical tube. See e.g. a short review in (Sharipov, 1996). It has already been shown, in such geometries, that working directly with the "Bhatnagar-Gross-Krook" (BGK) approximation of the kinetic equations (Loyalka, 1969) gives results for the "thermomolecular pressure difference" which are not too far from the Dusty-Gas Model predictions (Gupta and Storvick, 1970). However, no method has been reported for the precise numerical evaluation of thermal transpiration parameters in the rarefied regime for general porous media described by a $3 \mathrm{D}$ image.

The purposes of our research project are thus the following: 
- To derive a coherent change-of-scale formalism yielding macroscopic equations for non-isothermal pure gas transport in a porous medium, starting directly from kinetic equations, and to discuss it with respect to the Dusty-Gas Model and other formulations ;

- To design a tool allowing to compute effective properties for Knudsen transport, and for thermal transpiration in whatever porous medium, to test it and to examine its results, comparing them to other referenced works when possible.

After a first part where the essential physics are presented and the Dusty-Gas Model is discussed, we will present in this document a formulation for pure gas transport (that is, not including binary diffusion) in non-isothermal conditions as it appears from homogenization theory (Bensoussan et al., 1978; Bardos et al., 1997) when applied to the Maxwell-Boltzmann kinetic equation. The associated energy transport is also treated. The performed change of scale provides a set of macroscopic variables and equations, as well as a set of closure problems that are to be solved at microscopic scale. This theoretical model will be discussed with respect to the DGM theory. A companion article (Vignoles et al., 2007) will present a numerical implementation of this theory, validation tests and a discussion of the results.

\section{Phenomenology of gas transport and existing models}

\subsection{Dusty-Gas Model}

The DGM is based on the mass balance relations for $n+1$ species, of which the first $n$ are true gas molecules and the $(n+1)^{\text {th }}$ species (the "dust") has a huge molecular mass, a vanishing mole fraction, a uniform spatial distribution, and is held motionless. We restrict ourselves to the case where only one gas species is present.

\subsubsection{Flux-gradient relation}

The flux-gradient relation resulting from the analysis of (Mason and Malinauskas, 1983) follows from the Stefan-Maxwell multicomponent relationships where the dust and the gas are the two involved species. They may be presented under the following form :

$$
\frac{n_{d}\left(1-\Delta_{g d}\right)}{n D_{g d}}\left[\frac{\mathbf{J}}{C}+\frac{B}{\eta}(\nabla P-C \mathbf{F})\right]=
$$




$$
-\nabla \ln P+\mathbf{F} / \mathcal{R} T-\frac{n_{d}\left(\alpha_{g d}\right)_{t r}}{n+n_{d}} \nabla \ln T
$$

In this equation, the fluxes are on the left-hand side and the forces on the right-hand side. The Knudsen diffusion and thermal transpiration coefficients are identified as :

$$
\begin{gathered}
D_{K}=\frac{n D_{g d}}{n_{d}\left(1-\Delta_{g d}\right)} \\
\alpha_{T}=\frac{n_{d}\left(\alpha_{g d}\right)_{t r}}{n+n_{d}}
\end{gathered}
$$

In the dusty-gas limit, $n_{d}$ goes to zero, but so do the $D_{g d}$ and $\alpha_{g d}$ factors, in such a way that the defined coefficients do converge to some finite limit. One of the weaknesses of the original derivation is that no explicit dependance of the various gas-dust coefficients to the small parameter $n_{d}$ is given. Another unclear point is that nothing is said of the dust volume fraction $\phi_{d}$ when one takes the limit. Is it held constant ? The only answer to this question is the (implicit) assumption that all quantities, after taking the limit, are averaged over the whole space. By doing so, care has to be taken in order to reduce progressively the molecular mobility as the size grows ; this point has never been mentioned in the historical presentation of the DGM.

The extrinsic velocity (filtration velocity) $\mathbf{v}=\mathbf{J} / C$ obeys the following relation :

$$
\mathbf{v}=\epsilon \mathbf{u}=-\left(\frac{P B}{\eta}+D_{K}\right) \frac{\nabla P}{P}-\alpha_{T} D_{K} \frac{\nabla T}{T}
$$

and, by making use of the perfect gas law, one obtains the classical Darcy-Klinkenberg expression for mass fluxes.

The Dusty-Gas Model coefficients $D_{K}, B / \eta$, and $\alpha_{T}$ are evaluated from various dimensionless collision integrals. Let us discuss them in more detail.

\subsubsection{The viscous transport coefficient $B / \eta$}

Two contributions are evident in this term, one from the gas which is viscosity, and the other from the porous medium, which is permeability. Classical models are available for viscosity, either directly from kinetic theory (Chapman and Cowling, 1970; Hirschfelder et al., 1963), or from critical temperature and molar volume (Reid et al., 
1987). Pure gas viscosities almost do not depend on pressure, while they depend on the square root of the molecular mass and temperature. It is convenient for later use to introduce the notion of molecular mean free path $\bar{l}$, such that the kinematic viscosity $\eta / \rho$ be the product of the mean quadratic speed $\bar{c}=\sqrt{8 \mathcal{R} T / \pi M}$ and of $\bar{l}$ :

$$
\nu=\frac{\eta}{\rho}=\frac{1}{3} \bar{c} \bar{l}
$$

If the perfect gas law applies, then $\bar{l}$ is proportional to $T / P$.

The pore Knudsen number will taken as the ratio of this mean free path to the pore diameter :

$$
K n=\frac{\bar{l}}{d_{p}}=\frac{3 \eta}{\rho \bar{c} d_{p}}
$$

It is a measure of the degree of gaseous rarefaction.

The permeability may be obtained from a modified Stokes law for spheres or from a Poiseuille law for hollow cylinders. From the original formulation, one has :

$$
B=\frac{1}{R_{0} n_{d}}
$$

where $R_{0}$ is a geometrical factor proportional to the dust particle radius $r$, and $n_{d}$ varies as $r^{-3}$. Since no other details on the latter dependency is known, it is usually preferred to use the following expression (see e. g. (Tomadakis and Robertson, 2005)):

$$
B=\epsilon{\underline{\underline{\tau_{v}}}}^{-1} \frac{d_{p}^{2}}{32}
$$

where $\tau_{v}$ is a "tortuosity factor", i.e. a corrective factor usually larger than 1 which represents the precise effect of the pore geometry on viscous transport, and $d_{p}$ is an estimation of the mean pore hydraulic diameter (e.g. using Jäger's formula $4 \epsilon / \sigma_{v}$ for a generalized cylinder, $\sigma_{v}$ being the internal surface area in $\left.m^{-1}\right)$. The inclusion of the porosity inside formula (8) accounts for the law of mixtures (the fluid does not flow inside the solid parts), and the tensorial nature of the tortuosity factor accounts for the possible anisotropy of the porous medium. 
2.1.3. The Knudsen transport coefficient $D_{K}$

This coefficient is evaluated following the same principle as before, that is, the classical Chapman-Enskog relation is adapted to the case of a gas-dust interdiffusion pair. The resulting expression of $D_{K}$ is :

$$
D_{K}=\frac{3 \pi}{32} \bar{c} \cdot \frac{1}{n_{d} S_{d} \Omega_{g d}} \cdot \frac{1}{1-\left|\alpha_{T}\right| / 5}
$$

The $\frac{1}{n_{d} S_{d}}$ term represents the influence of the porous medium $\left(S_{d}\right.$ varies with $r^{2}$ and $n_{d}$ with $r^{-3}$ ), while the $\Omega_{g d}{ }^{-1}$ term accounts for the precise kind of gas-dust collision through the collision integral. $\Omega_{g d}$ is unity for elastic specular collisions, $\frac{13}{9}$ for elastic diffuse collisions (obeying Knudsen's cosine law), and $1+\frac{\pi}{8}$ for inelastic scattering (i.e. also obeying Knudsen's law but with temperature accommodation). Other more sophisticated models exist when there are gas-wall interactions before contact. The last term in equation (9) is a secondorder correction depending moderately on pressure, and features the thermal transpiration parameter $\alpha_{T}$ :

$$
\frac{1}{1-\Delta_{g d}}=\frac{1}{1-\left|\alpha_{T}\right| / 5}=\frac{P+\pi_{g}}{P+\pi_{g}^{\prime}}
$$

Instead of evaluating the coefficients $\pi_{g}$ and $\pi_{g}^{\prime}$ through the dusty-gas limit approach, a simpler approximation is proposed (Mason et al., 1967) :

$$
\pi_{g}=\frac{2}{5} \frac{T\left(\lambda_{i i}\right)_{t r}}{\left[D_{K}\right]_{1}} \text { and } \pi_{g}^{\prime} \approx 0.9 \pi_{g}
$$

where $\left(\lambda_{i i}\right)_{t r}$ is the translational component of the gas thermal conductivity :

$$
\left(\lambda_{i i}\right)_{t r}=\frac{15}{4} \frac{\mathcal{R}}{M} \eta
$$

and $\left[D_{K}\right]_{1}$ is the Knudsen diffusion coefficient without pressure correction.

Again, it is usually found convenient to reformulate $D_{K}$ into a form where the pore geometrical quantities appear explicitly :

$$
D_{K}=\frac{\epsilon}{3} \bar{c} d_{p} \underline{\underline{\tau_{K}}}{ }^{-1} \frac{P+\pi_{g}}{P+\pi_{g}^{\prime}} \xi
$$

Note that the precise contribution of the porous medium geometry appears, as for permeability, through the law of mixtures and the 
presence of a tensorial tortuosity factor. The value of $\tau_{K}$ for a cylindrical pore is 1 . The factor $\xi=\Omega_{g d}^{-1}$ accounts for the precise type of molecule-wall collisions.

The pressure dependency factor may be recast into a more suitable form, neglecting the tortuosity factor :

$$
\frac{P+\pi_{g}}{P+\pi_{g}^{\prime}}=\frac{2 / 3+K n}{2 / 3+0.9 K n}
$$

Collecting together the viscous and Knudsen contributions to mass transport, one gets the evolution of its contribution between continuum and free-molecule limits, as a function of the Knudsen number

$$
\frac{P B}{\eta}+D_{K}=\epsilon \bar{c} d_{p}\left(\frac{3 \pi}{256} \underline{\underline{\tau_{v}^{-1}}} K n^{-1}+\frac{1}{3} \underline{\underline{\tau_{K}^{-1}}} \frac{A_{g}+K n}{A_{g}+0.9 K n} \xi\right)
$$

where $A_{g}$ is of order $2 / 3$.

It is interesting to note that the dimensionless ratio of Knudsen flux to viscous flux is, in a straight pore (Kerkhof, 1997; Froment and Bischoff, 1979) :

$$
K e_{2}=1 / N_{D i N}=\frac{\eta D_{K}}{P B}=\frac{256}{9 \pi} \frac{\tau_{v}}{\tau_{K}} K n \frac{A_{g}+K n}{A_{g}+0.9 K n} \xi
$$

It is another measure of the rarefaction degree, which differs from the Knudsen number by approximately one order of magnitude - that is, the ratio $K e_{2}$ between slip-flow and Darcy velocities is unity when the Knudsen number is about $1 / 10$.

\subsubsection{The thermal transpiration factor $\alpha_{T}$}

As mentioned briefly before, the authors of the DGM did not use the exact approach that they have built up, but used rather a simplified estimation, based on the fact that the inverses of the thermal diffusion factors are linear in the mole fraction. The DGM estimation of the thermal diffusion factor is then :

$$
\left|\alpha_{T}^{-1}\right|=2+\frac{5 \mathcal{R} \rho\left[D_{K}\right]_{1}}{M\left(\lambda_{i i}\right)_{t r}}
$$

The sign of $\alpha_{T}$ is indeed negative, which means that the gas has tendency to migrate towards the hotter side of the medium. 
Developing all coefficients in the preceding expression yields an explicit dependence of $\alpha_{T}$ on temperature, pressure, and porous medium related quantities :

$$
-\alpha_{T}^{-1}=2+\frac{8 P}{5 \mathcal{R} T}\left(\pi \sigma_{c}^{2} \Omega_{v}\right) \cdot \epsilon d_{p} \tau_{K}^{-1} \xi
$$

The tensorial character of the transport coefficient has been dropped here since it is not present in the hypotheses that have been used for the derivation of such an approximate expression. Neglecting any contribution from the precise geometry (porosity, tortuosity) the following approximate relation should hold :

$$
-\alpha_{T}^{-1}=2+\frac{4}{3} \xi K n^{-1}
$$

Some remarks have to be made :

- The low-pressure limit for $\alpha_{T}$ should be close to $-1 / 2$.

- The high-pressure limit should be zero $\left(\alpha_{T} \propto K n\right)$.

The proposed evaluation of $\alpha_{T}$ does not take into account the precise knowledge of the porous medium geometry. In particular, it is not told in detail whether the factor 2 in eqn. (18) should be altered by a tensorial quantity representing the anisotropy of the pores. Indeed, this is one of the weaknesses of the Dusty-Gas Model in itself, for which the geometrical factor computation is left to posterior identification to the user of the theory. Indeed, the authors report that the best way to use the model in practice is to consider the number 2 in eq. (19) as an adjustable parameter, as well as $\pi_{g}$ and $\pi_{g}^{\prime}$ in eq. (10). The necessity for this adjustment arises from several factors : first, as mentioned, the model does not account for geometrical effects ; second, the model has been compared to experiments in which some extra effects like adsorption/desorption in micropores (Angstrom to nanometer sizes) can play a role in the rarefied limit.

\subsection{Binary Friction Model}

The original presentation of the Binary Friction Model (Kerkhof, 1996) is made in an isothermal hypothesis, however, guidelines are given for the rather straightforward incorporation of thermal transpiration, through a thermal-creep contribution to the frictional forces. In the case of a pure gas, both models are expected to yield equal 
formal results. The formalism proposed by Kerkhof does not provide clues for the identification of the transport coefficient from kinetictheory level quantities such as collision integrals ; in addition to the formal equivalence to the DGM in the pure-gas case, this is a sufficient reason for not describing it here in details.

\subsection{Results From the Resolution of Kinetic EQUATIONS IN CYLINDRICAL PORES}

In parallel with the attempts to extract a macroscopic formalism from kinetic-theory considerations, much work has been done on the direct resolution of the kinetic equations in a straight pore. The Bhatnagar-Gross-Krook (BGK) approximation has been used (Loyalka, 1969), and more recently the $s$-model (Sharipov, 1996). The attention has been brought on the precise molecule-wall interaction, which may be partially diffuse and partially purely elastic, and on the amount of thermal accommodation between wall and molecules.

It appears from such works that the relationships obtained through the evaluation of the moments are similar to the DGM/BFM formalisms, and the slip (Knudsen diffusion) and creep (thermal transpiration) coefficients have been evaluated numerically. The gradient is taken only along the pore length. No account of porosity is made.

These results, as well as the DGM predictions, will be used as a benchmark for the approach that is going to be developed in the following parts.

\section{Homogenization of Maxwell-Boltzmann's equations in a porous medium for a single gas}

The present derivation of mass and heat transfer for a single gas in a porous medium has been presented in a more exhaustive fashion in (Charrier and Dubroca, 2003a; Charrier and Dubroca, 2003b). We will recall the main results in the following sections. The considered gas-wall collisions will be partially elastic, and partially diffuse with thermal accommodation, according to an accommodation factor $\sigma=$ $(8 / \pi)\left(\xi^{-1}-1\right)$ ranging between 0 and 1 . 


\subsection{LOCAL PROBLEM FORMULATION}

Let us denote $\Omega$, the open set in $\mathbb{R}^{3}$ occupied by the porous material, $\Omega_{s} \subset \Omega$ the open subset of $\Omega$ occupied by the solid phase, $\Omega_{f}$ the open subset occupied by the gas and $\Sigma$ the interface between $\Omega_{f}$ et $\Omega_{s}$. In all the following $\mathbf{n}=\mathbf{n}(\mathbf{x}), \mathbf{x} \in \Sigma$, denotes the normal to $\Sigma$ at point $\mathbf{x}$, outgoing from $\Omega_{s}$.

The solid phase is characterized by its density $\rho_{s}$, its specific heat at constant volume $C_{v}$ and its thermal conductivity $\lambda_{s}$. These quantities are assumed to be known and constant. We assume also that the mechanical deformations in the solid can be neglected and that only the thermal energy is evolving. Hence the only unknown in the solid phase is the temperature field $T(\mathbf{x}, t)$ defined on $\Omega_{s}$ whose evolution is given by the classical heat equation

$$
\rho_{s} C_{v} \partial_{t} T-\operatorname{div}_{x}\left(\lambda_{s} \nabla T\right)=0 .
$$

The gas will be described by a kinetic model. We denote $f=$ $f(\mathbf{x}, t, \mathbf{c})$ the mass distribution function and the evolution of the gas is given by the Boltzmann equation in $\Omega_{f}$

$$
\partial_{t} f+\mathbf{c} \cdot \nabla_{x} f=Q(f, f),
$$

The collision operator $Q$ is defined by

$$
Q(f, f)(\mathbf{v})=\rho^{-1} \int_{\mathbb{R}_{w}^{3}} \int_{S^{2}}\left[f\left(\mathbf{c}^{\prime}\right) f\left(\mathbf{d}^{\prime}\right)-f(\mathbf{c}) f(\mathbf{d})\right] b(\mathbf{c}-\mathbf{d}, \omega) d \mathbf{d} d \omega
$$

where $\mathbf{c}^{\prime}$ and $\mathbf{d}^{\prime}$ are given by

$$
\begin{aligned}
& \mathbf{c}^{\prime}=\mathbf{c}-[(\mathbf{c}-\mathbf{d}) \cdot \omega] \omega, \\
& \mathbf{d}^{\prime}=\mathbf{d}-[(\mathbf{d}-\mathbf{c}) \cdot \omega] \omega,
\end{aligned}
$$

and where the scattering kernel $b(\mathbf{z}, \omega)=|z| \Sigma_{c}(|z|, \cos (\mathbf{z}, \omega))$ depends on the interaction potential between molecules which is considered and the cross-section $\Sigma_{c}$ is given by (see (Cercignani, 1987))

$$
\begin{aligned}
\Sigma_{c}(z, \cos \theta)= & r^{2} \cos \theta \\
& (\text { hard sphere potential) } \\
\Sigma_{c}(z, \cos \theta)= & r^{2}|z|^{\kappa-1} \cos \theta, \quad \kappa \in[0,1[
\end{aligned}
$$

(variable hard sphere potential) 
In any case, the collision term satisfies the following fundamental properties ensuring conservation laws and $\mathrm{H}$-theorem

$$
\begin{aligned}
\int_{\mathbb{R}_{c}^{3}} Q(f, f) \psi(\mathbf{c}) d \mathbf{c} & =0, \forall \psi(\mathbf{c})=\alpha+\beta \cdot \mathbf{c}+\gamma|c|^{2}, \\
\int_{\mathbb{R}_{c}^{3}} Q(f, f) \ln (f) d \mathbf{c} \leq 0, & \leq \Leftrightarrow Q(f, f)=0 \\
\int_{\mathbb{R}_{c}^{3}} Q(f, f) \ln (f) d \mathbf{c} & =0 \Leftrightarrow \exp \left(\alpha+\beta \cdot \mathbf{c}+\gamma|c|^{2}\right) \\
Q(f, f) & =0 \Leftrightarrow f=
\end{aligned}
$$

Macroscopic quantities (density, momentum, total energy) are defined by

$$
\rho=<f>, \quad \rho \mathbf{u}=<f \mathbf{c}>, \quad \rho E=<f \frac{1}{2}|c|^{2}>
$$

where $\langle\psi\rangle=\int_{\mathbb{R}_{c}^{3}} \psi(\mathbf{c}) d \mathbf{c}$ is the velocity space averaging operator.

The particles bouncing back from a wall collision result partly from a specular reflection and from a diffuse reflection. Let $\sigma$ be the accommodation factor, that is, the fraction of diffusely reflected particles. Then, the distribution of particles outgoing from the interface $\Sigma$ obeys the following expression :

$$
\begin{gathered}
\left.f(\mathbf{x}, \mathbf{c})\right|_{\mathbf{x} \in \Sigma, \mathbf{c} \cdot \mathbf{n}(\mathbf{x})>0}=\sigma\left(\int_{\mathbf{w} \cdot \mathbf{n}<0} \mathbf{w} \cdot \mathbf{n}(\mathbf{x}) \mid f(\mathbf{x}, \mathbf{w}) d \mathbf{w}\right) M_{n}(T(\mathbf{x}))(\mathbf{c}) \\
+(1-\sigma) f(\mathbf{x}, \mathbf{c}-2 \mathbf{c} \cdot \mathbf{n} \mathbf{n})
\end{gathered}
$$

where $M_{n}(T)$ is a Maxwellian distribution with an average velocity equal to zero and temperature $T$, normalized by the condition

$$
\int_{\mathbf{c} \cdot \mathbf{n}>0}(\mathbf{c} \cdot \mathbf{n}) M_{n}(T)(\mathbf{c}) d \mathbf{c}=1
$$

, so that $M_{n}(T)$ is given by :

$$
M_{n}(T)(\mathbf{c})=\frac{1}{2 \pi(\mathcal{R} T / M)^{2}} \exp \left(-\frac{|c|^{2}}{2 \mathcal{R} T / M}\right) .
$$

The continuity of the energy flux on $\Sigma$ takes the following form :

$$
\int_{\mathbb{R}_{c}^{3}} f(\mathbf{x}, \mathbf{c}) \frac{|c|^{2}}{2}(\mathbf{c} \cdot \mathbf{n}(\mathbf{x})) d \mathbf{c}+\lambda \nabla_{x} T(\mathbf{x}) \cdot \mathbf{n}(\mathbf{x})=0, \mathbf{x} \in \Sigma,
$$


Finally the microscopic model we will consider writes

$$
\begin{aligned}
\partial_{t} f+\mathbf{c} \cdot \nabla_{x} f= & Q(f, f), \mathbf{x} \in \Omega_{f} \\
\rho C_{v} \partial_{t} T= & \left(\operatorname{div}_{x} \lambda_{s} \nabla_{x} T\right), \mathbf{x} \in \Omega_{s}, \\
\left.f(\mathbf{x}, \mathbf{c})\right|_{\mathbf{c} \cdot \mathbf{n}>0}= & \sigma\left(\int_{\mathbf{w} \cdot \mathbf{n}<0}|\mathbf{w} \cdot \mathbf{n}| f(\mathbf{x}, \mathbf{w}) d \mathbf{w}\right) M_{n}(T) \\
& +(1-\sigma) f(\mathbf{x}, \mathbf{c}-2 \mathbf{c} \cdot \mathbf{n} \mathbf{n}), \mathbf{x} \in \Sigma, \\
\lambda_{s} \nabla_{x} T \cdot \mathbf{n}= & -\int \frac{1}{2}|c|^{2} f(\mathbf{x}, \mathbf{c}) \mathbf{c} \cdot \mathbf{n} d \mathbf{c}, \mathbf{x} \in \Sigma,
\end{aligned}
$$

Such a model obeys the following properties :

- Global conservation of mass and energy are ensured

- The H-theorem is verified, that is, the local entropy production is positive (under the convention used by physicists).

\subsection{Homogenization}

\subsubsection{Space decoupling}

It will now be assumed that the space variable may decoupled into two contributions, differing by their scale :

$$
\mathbf{x}=\mathbf{x}^{\prime}+\mathbf{y}
$$

where $\mathbf{x}^{\prime}$ has broad variations (i.e. $\psi\left(\mathbf{x}^{\prime}\right)$ is a local average of $\psi(\mathbf{x})$ ) and $\mathbf{y}$ has short-scale variations (i.e. $\psi(\mathbf{y})$ is a local perturbation of $\psi(\mathbf{x})$ with respect to $\left.\psi\left(\mathbf{x}^{\prime}\right)\right)$. Let us define an integration support $Y \subset \Omega$ for $\mathbf{y}$, which is a small space region, split into two subsets $Y_{s}$ and $Y_{f}$ with share a common frontier $\partial Y_{f s}$. The ratio $\varepsilon=x / y$ is a small parameter allowing to use the tools of asymptotic analysis. By taking the limit $\varepsilon \rightarrow 0$, one simultaneously changes scale in space and takes a hydrodynamic limit for the fluid.

It is here considered that the fluid and the solid are in local equilibrium, so that only one temperature defined on $\Omega$ is enough to describe the system.

Reference quantities will now be chosen in order to put an emphasis on the change-of-scale procedure :

- A reference length $L_{*}$ of the order of $|Y|^{1 / 3}$, that is, a macroscopic length. The parameter $\varepsilon$ is $d_{p} / L_{*}$. 
- A Knudsen number $K n_{*}$ relative to the macroscopic length $L_{*}$ is introduced ; note that $K n_{*}=\varepsilon K n$.

- The reference temperature $T_{*}$ depends on the precise application chosen.

- The reference velocity $u_{*}$ is based on the convection velocity. Thus the Mach number is $M a_{*}=u_{*} / a_{*}$, where $a_{*}=\sqrt{\gamma \mathcal{R} T / M}$ and $\gamma=\frac{C_{p, g}}{C_{v, g}}$. Note that $a_{*}$ is very close to $\bar{c}$ since $\gamma$ is close to unity (e.g. $5 / 3$ for simple gases).

- The reference quantity for the distribution function $f$ is $f_{*}=$ $\rho_{*} / a_{*}^{3}$.

- The Reynolds number is $R e_{*}=\frac{\rho_{*} u_{*} L_{*}}{\eta_{*}}$. Note that $R e_{*}=\frac{\gamma M a_{*}}{K n_{*}}$.

- The Prandtl number is $\operatorname{Pr}_{*}=\frac{C_{p, *} \eta_{*}}{\lambda_{*}}$ and is of order unity, a classical fact for gases.

All quantities may be split into a reference and a dimensionless factor $: \psi=\psi_{*} \tilde{\psi}$. By doing so, the microscopic problem may be recast in the following form :

$$
\begin{aligned}
& M a_{*} \partial_{\tilde{t}} \tilde{f}+\tilde{\mathbf{c}} \cdot \nabla_{\tilde{\mathbf{x}}} \tilde{f}=\frac{1}{K n_{*}} \tilde{Q}(\tilde{f}, \tilde{f}), \tilde{\mathbf{x}} \in \Omega(f, 40) \\
& \left.\left(\frac{R e_{*} P r_{*}}{\gamma} \frac{\mathrm{a}_{*, g}}{\mathrm{a}_{*, s}}\right) \tilde{\rho} \tilde{C}_{v} \partial_{\tilde{t}} \tilde{T}=\operatorname{div}_{\tilde{x}}\left(\tilde{\lambda}_{s} \nabla_{\tilde{x}} \tilde{T}\right), \tilde{\mathbf{x}} \in \Omega_{\S}, 41\right) \\
& \sigma\left(\int_{\tilde{\mathbf{w}} \cdot \mathbf{n}<0}|\tilde{\mathbf{w}} \cdot \mathbf{n}| \tilde{f} d \tilde{\mathbf{w}}\right) \tilde{M}(\tilde{T}) \ldots \\
& \ldots+(1-\sigma) \tilde{f}(\tilde{\mathbf{x}}, \tilde{\mathbf{c}}-2 \tilde{\mathbf{c}} \cdot \mathbf{n} \mathbf{n})=\tilde{f}_{\mid \tilde{\mathbf{c}} . \mathbf{n}>0}, \tilde{\mathbf{x}} \in \Sigma, \\
& -\left(\frac{\gamma(\gamma-1) P r_{*}}{K n_{*}} \frac{\lambda_{*, g}}{\lambda_{*, s}}\right) \int \frac{1}{2}|\tilde{c}|^{2} \tilde{f} \tilde{\mathbf{c}} \cdot \mathbf{n} d \tilde{\mathbf{c}}=\tilde{\lambda}_{s} \nabla_{\tilde{x}} \tilde{T} \cdot \mathbf{n}, \tilde{\mathbf{x}} \in \Sigma,
\end{aligned}
$$

where $\mathrm{a}$ is the the thermal diffusivity $\frac{\lambda}{\rho C_{p}}$. In the following, all tildes will be dropped out for sake of simplicity.

\subsubsection{Identified scalings}

The identified scalings are : 
- $M a_{*} \approx \varepsilon$, i.e., the flow is subsonic. This implies that compressibility effects are small in the presented study. Neglecting them represents a safe approximation in numerous practical cases (Haddad et al., 2007)

- $K n_{*} \approx \varepsilon$, i.e. the gas is in a transition regime,

- As a consequence, $R e=\frac{\gamma M a_{*}}{K n_{*}}=\mathcal{O}(1)$, so the flow is in a laminar regime.

- In the considered applications (temperatures around $1000 K$ and pressures from 1 to $100 \mathrm{kPa}$ ), the thermal conductivity ratio $\frac{\lambda_{*, g}}{\lambda_{*, s}}$ is small, and scales as $\varepsilon$.

- Since the density ratio $\frac{\rho_{*, g}}{\rho *, s}$ also scales as $\varepsilon$ and the mass heat capacity ratio is of order unity, the thermal diffusivity ratio is expected to be unity. Thus, $\left(\frac{R e_{*} P r_{*}}{\gamma} \frac{\mathrm{a}_{*, g}}{\mathrm{a}_{*, s}}\right)$ in eq. (41) is also of order unity.

- In eq. (43), the term $\left(\frac{\gamma(\gamma-1) P r_{*}}{K n_{*}} \frac{\lambda_{*, g}}{\lambda_{*, s}}\right) \approx \varepsilon^{-1}$, that is, there is a weak coupling between the gas and the solid.

The scaled equations may be now rewritten as :

$$
\begin{aligned}
\varepsilon \partial_{t} f+\mathbf{c} \cdot \nabla_{x} f= & \frac{1}{\varepsilon} Q(f, f), \mathbf{x} \in \Omega_{f}, \\
\rho C_{v} \partial_{t} T= & \operatorname{div}_{x}\left(\lambda_{s} \nabla_{x} T\right), \mathbf{x} \in \Omega_{s}, \\
\left.f\right|_{\mathbf{c} \cdot \mathbf{n}>0}= & \sigma\left(\int_{\mathbf{w} \cdot \mathbf{n}<0}|\mathbf{w} \cdot \mathbf{n}| f d \mathbf{w}\right) M_{n}(T) \\
& +(1-\sigma) f(\mathbf{x}, \mathbf{c}-2 \mathbf{c} \cdot \mathbf{n} \mathbf{n}), \mathbf{x} \in \Sigma, \\
\lambda_{s} \nabla_{x} T \cdot \mathbf{n}= & -\int \frac{1}{2}|c|^{2} f \mathbf{c} \cdot \mathbf{n} d \mathbf{c}, \mathbf{x} \in \Sigma,
\end{aligned}
$$

\subsubsection{Expansion of the variables}

We look for an expansion of $T(\mathbf{x})$ and $f(\mathbf{x})$ in successive powers of $\varepsilon$

$$
\begin{aligned}
T^{\varepsilon}\left(\mathbf{x}^{\prime}, \mathbf{y}\right)_{\mid y=x^{\prime} / \varepsilon} & =T_{0}^{\varepsilon}\left(\mathbf{x}^{\prime}, \mathbf{y}\right)+\varepsilon T_{1}^{\varepsilon}\left(\mathbf{x}^{\prime}, \mathbf{y}\right)+\varepsilon^{2} T_{2}^{\varepsilon}\left(\mathbf{x}^{\prime}, \mathbf{y}\right)+\ldots, \\
f^{\varepsilon}\left(\mathbf{x}^{\prime}, \mathbf{y}, \mathbf{c}\right)_{\mid y=x^{\prime} / \varepsilon} & =f_{0}^{\varepsilon}\left(\mathbf{x}^{\prime}, \mathbf{y}, \mathbf{c}\right)+\varepsilon f_{1}^{\varepsilon}\left(\mathbf{x}^{\prime}, \mathbf{y}, \mathbf{c}\right)+\varepsilon^{2} f_{2}^{\varepsilon}\left(\mathbf{x}^{\prime}, \mathbf{y}, \mathbf{c}\right) .
\end{aligned}
$$

Inserting these expressions into eqs. (44-47) and collecting like powers of $\varepsilon$ together leads to the following relations : 
Order 0:

$$
\begin{aligned}
-\operatorname{div}_{y}\left(\lambda_{s} \nabla_{y} T_{0}\right)= & 0, \mathbf{y} \in Y_{f} \\
\mathbf{c} \cdot \nabla_{y} f_{0}= & Q\left(f_{0}, f_{0}\right), \mathbf{y} \in Y_{f}, \\
\left.f_{0}(\mathbf{y}, \mathbf{c})\right|_{\mathbf{c} \cdot \mathbf{n}>0}= & \sigma\left(\int_{\mathbf{w} \cdot n<0}|\mathbf{w} \cdot \mathbf{n}| f_{0} d \mathbf{w}\right) M_{n}\left(T_{0}\right) \\
& +(1-\sigma) f_{0}(\mathbf{y}, \mathbf{c}-2 \mathbf{c} \cdot \mathbf{n} \mathbf{n}), \mathbf{y} \in \partial Y_{f s} \\
\lambda_{s} \nabla_{y} T_{0} \cdot \mathbf{n}= & 0, \mathbf{y} \in \partial Y_{f s},
\end{aligned}
$$

Order 1:

$$
\begin{aligned}
-\operatorname{div}_{y}\left(\lambda_{s} \nabla_{y} T_{1}\right) & =\operatorname{div}_{y}\left(\lambda_{s} \nabla_{x^{\prime}} T_{0}\right)+\operatorname{div}_{x^{\prime}}\left(\lambda_{s} \nabla_{y} T_{0}\right) \\
L\left(f_{1}\right)-\mathbf{c} \cdot \nabla_{y} f_{1} & =\mathbf{c} \cdot \nabla_{x^{\prime}} f_{0} \\
\left.f_{1}\right|_{\mathbf{c} \cdot \mathbf{n}>0} & =\sigma\left(\int_{\mathbf{w} \cdot \mathbf{n}<0}|\mathbf{w} \cdot \mathbf{n}| f_{0} d \mathbf{w}\right) \frac{d}{d T} M_{n}\left(T_{0}\right) T_{1} \\
& +\sigma\left(\int_{\mathbf{w} \cdot \mathbf{n}<0}|\mathbf{w} \cdot \mathbf{n}| f_{1} d \mathbf{w}\right) M_{n}\left(T_{0}\right) \\
& +(1-\sigma) f_{1}(\mathbf{x}, \mathbf{c}-2 \mathbf{c} \cdot \mathbf{n} \mathbf{n}), \mathbf{y} \in \Sigma \\
\lambda_{s} \nabla_{y} T_{1} \cdot \mathbf{n} & =-\lambda_{s} \nabla_{x} T_{0} \cdot \mathbf{n}-\int \frac{1}{2}|c|^{2} f_{0} \mathbf{c} \cdot \mathbf{n} d \mathbf{c}, \mathbf{y} \in \Sigma,
\end{aligned}
$$

where $L$ is the linearized collision operator defined by

$$
L(g)=L_{f_{0}}(g)=Q\left(f_{0}, g\right)+Q\left(g, f_{0}\right) .
$$

Order 2:

$$
\begin{aligned}
\rho_{s} C_{v} \partial_{t} T_{0}= & \operatorname{div}_{x^{\prime}}\left(\lambda_{s}\left(\nabla_{x^{\prime}} T_{0}+\nabla_{y} T_{1}\right)\right) \\
& +\operatorname{div}_{y}\left(\lambda_{s}\left(\nabla_{x^{\prime}} T_{1}+\nabla_{y} T_{2}\right)\right), \\
\partial_{t} f_{0}+\mathbf{c} \cdot \nabla_{x^{\prime}} f_{1}= & Q\left(f_{1}, f_{1}\right)+L\left(f_{2}\right)-\mathbf{c} \cdot \nabla_{y} f_{2}, \\
\lambda_{s}\left(\nabla_{x^{\prime}} T_{1}+\nabla_{y} T_{2}\right) \cdot \mathbf{n}= & -\int f_{1} \frac{1}{2}|c|^{2}(\mathbf{c} \cdot \mathbf{n}) d \mathbf{c}, \mathbf{y} \in \Sigma .
\end{aligned}
$$

\subsubsection{Closure and auxiliary problems}

An important result is that $f_{0}$ is an absolute Maxwellian distribution, independent of $\mathbf{y}$, with temperature $T_{0}$; its average over velocity space is the zeroth-order approximation for the density $\rho_{0}$. Thus, $\nabla_{x^{\prime}} f_{0} / f_{0}$ can be written as a linear combination of $\nabla_{x^{\prime}} T_{0}$ and $\nabla_{x^{\prime}} \rho_{0}$.

On the other hand, the coupling between $T$ and $f$ is weak. Physically, this means that the solid phase, being much more conductive 
than the fluid phase with respect to heat, ensures by itself the buildup of the thermal gradient, which is then communicated to the fluid. So there is no effect of $\rho$ on $T$ to be taken into account.

It is then justified to seek $f_{1}$ and $T_{1}$ in the form :

$$
\begin{aligned}
& f_{1}=-f_{0}\left(\alpha \cdot \nabla_{x^{\prime}} T_{0}+\beta \cdot \nabla_{x^{\prime}} \rho_{0}\right) \\
& T_{1}=\gamma \cdot \nabla_{x^{\prime}} T_{0}
\end{aligned}
$$

Let $L$ be a linearized collision operator. It may be deduced directly from the Boltzmann collision operator $Q$ :

$$
L(f)=Q\left(f, f_{0}\right)+Q\left(f_{0}, f\right)
$$

Alternately, it may be given by models. In the BGK approximation (Loyalka, 1969), the linearized operator $L$ is evaluated analytically. Indeed, it has the form :

$$
L(f)=\left.f \frac{d}{d f}\left(\frac{1}{\tau}[M(f)-f]\right)\right|_{f=f_{0}}
$$

After some algebra, the final form of $L(f)$ is :

$$
L(f)=\frac{1}{\tau}\left(\frac{f_{0}}{\rho_{0}}\left(\begin{array}{c}
\frac{5}{2}-\frac{M|c|^{2}}{2 \mathcal{R} T_{0}} \\
\frac{M \mathbf{c}}{\mathcal{R} T_{0}} \\
\frac{M}{\mathcal{R} T_{0}}\left(\frac{M|c|^{2}}{3 \mathcal{R} T_{0}}-1\right)
\end{array}\right) \cdot\left(\begin{array}{c}
\rho \\
\rho \mathbf{u} \\
\rho E
\end{array}\right)-f\right)
$$

The same work has been performed on the Ellipsoidal Statistical (ES-BGK) model, for which $E$ in eq. (66) is replaced by $\underline{\underline{\mathcal{E}}}=$ $\frac{1}{2 \rho}\langle f \mathbf{c} \otimes \mathbf{c}\rangle$, and $M(f)$ in eq. (65) is replaced by :

$$
G(f)=\frac{\rho}{\sqrt{\operatorname{det}(2 \pi \underline{\underline{\mathcal{T}}})}} \exp \left(\frac{1}{2}(\mathbf{v}-\mathbf{u})^{T} \underline{\underline{\mathcal{T}}}^{-1}(\mathbf{v}-\mathbf{u})\right)
$$

where $\underline{\underline{\mathcal{I}}}=\frac{\mathcal{R} T}{M}(1-\nu) \underline{\underline{\mathrm{Id}}}+\nu \underline{\underline{\Theta}}$, and $\underline{\underline{\Theta}}=2 \underline{\underline{\mathcal{E}}}-\rho \mathbf{u} \otimes \mathbf{u}$. The coefficient $\nu$ allows to adjust the Prandtl number that stems out of the model. In the monatomic gas case, the correct choice for $\nu$ is $-1 / 2$.

In the following, the exact form of $L(f)$ will only be of concern when one goes to the numerical computations. The vectorial closure variables $\alpha=\left(\alpha_{i}(\mathbf{y}, \mathbf{c})\right)_{i=1,2,3}, \beta=\left(\beta_{i}(\mathbf{y}, \mathbf{c})\right)_{i=1,2,3}$, and $\gamma=$ $\left(\gamma_{i}(\mathbf{y}, \mathbf{c})\right)_{i=1,2,3}$ are solutions of auxiliary problems defined in the elementary cell $Y$ : 


$$
\begin{aligned}
& \left\{\begin{array}{l}
-L\left(f_{0} \alpha_{i}\right)+f_{0} \mathbf{c} \cdot \nabla_{y} \alpha_{i}=-f_{0} c_{i}\left(-\frac{3}{2 T_{0}}+\frac{M|c|^{2}}{2 \mathcal{R} T_{0}^{2}}\right), \text { in } Y_{f} \\
\alpha_{i} f_{0}-\sigma\left(-\frac{2}{T_{0}}+\frac{M|c|^{2}}{2 \mathcal{R} T_{0}^{2}}\right) \gamma_{i}=\sigma \int_{\mathbf{w} \cdot \mathbf{n}<0}|\mathbf{w}| \alpha_{i} f_{0} M_{n}\left(T_{0}\right) d \mathbf{w} \ldots \\
\ldots+(1-\sigma) \alpha_{i}(\mathbf{y}, \mathbf{c}-2 \mathbf{c} \cdot \mathbf{n} \mathbf{n}) f_{0}(\mathbf{y}, \mathbf{c}-2 \mathbf{c} \cdot \mathbf{n} \mathbf{n}), \\
\operatorname{in} \partial Y_{f s}, \text { and } \forall \mathbf{c} \cdot \mathbf{n}>0
\end{array}\right. \\
& \left\{\begin{array}{l}
-L\left(f_{0} \beta_{i}\right)+\mathbf{c} \cdot \nabla_{y}\left(f_{0} \beta_{i}\right)=-\frac{f_{0}}{\rho_{0}} c_{i}, \text { in } Y_{f} \\
\beta_{i}=\sigma \int_{\mathbf{w} \cdot \mathbf{n}<0}|\mathbf{w} \cdot \mathbf{n}| \beta_{i} M_{n}\left(T_{0}\right) d \mathbf{w} \ldots \\
\ldots+(1-\sigma) \beta_{i}(\mathbf{y}, \mathbf{c}-2 \mathbf{c} \cdot \mathbf{n} \mathbf{n}), \text { in } \partial Y_{f s}, \text { and } \forall \mathbf{c} \cdot \mathbf{n}>0
\end{array}\right.
\end{aligned}
$$

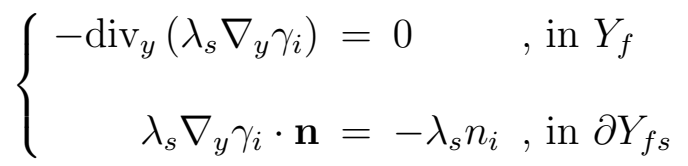

Eq. (68) may be somewhat simplified taking into account the expression of $f_{0}$ (eq.(52) and $f_{1}$ (eq. (56) :

$$
\left\{\begin{array}{l}
-L\left(f_{0} \alpha_{i}\right)+f_{0} \mathbf{c} \cdot \nabla_{y} \alpha_{i}=-f_{0} c_{i}\left(-\frac{3}{2 T_{0}}+\frac{M|c|^{2}}{2 \mathcal{R} T_{0}^{2}}\right), \text { in } Y_{f} \\
\alpha_{i}-\sigma\left(-\frac{2}{T_{0}}+\frac{M|c|^{2}}{2 \mathcal{R} T_{0}^{2}}\right) \gamma_{i}=\sigma \int_{\mathbf{w} \cdot \mathbf{n}<0}|\mathbf{n}| \alpha_{i} M_{n}\left(T_{0}\right) d \mathbf{w} \ldots \\
\ldots+(1-\sigma) \alpha_{i}(\mathbf{y}, \mathbf{c}-2 \mathbf{c} \cdot \mathbf{n} \mathbf{n}), \text { in } \partial Y_{f s}, \text { and } \forall \mathbf{c} \cdot \mathbf{n}>0
\end{array}\right.
$$

\subsubsection{Averaging and macroscopic relations}

On a macroscopic scale, a description is sought only for the macroscopic variables ; accordingly, one has to simultaneously integrate in velocity space and perform an average on the local support $Y$. The asymptotic transport model takes then the following form :

$$
\begin{array}{r}
\epsilon \partial_{t} \rho_{0}-\operatorname{div}_{x}\left(\underline{\underline{D}} \nabla_{x} \rho_{0}\right)-\operatorname{div}_{x}\left(\underline{\underline{\tilde{D}}} \nabla_{x} T_{0}\right)=0 \\
\rho_{s} C_{v, s} \partial_{t} T_{0}-\operatorname{div}_{x}\left(\underline{\underline{K}} \nabla_{x} T_{0}\right)=0
\end{array}
$$


where the tensorial effective coefficients are built with the closure variables obtained from problems $(71,69,70)$ in the following way :

$$
\begin{gathered}
\underline{\underline{D}}=\left\langle-f_{0} \mathbf{c} \otimes[\beta]_{Y_{f}}\right\rangle \\
\underline{\underline{\tilde{D}}}=\left\langle-f_{0} \mathbf{c} \otimes[\alpha]_{Y_{f}}\right\rangle \\
\underline{\underline{K}}=\left[\lambda_{s}\left(\underline{\underline{\mathrm{Id}}}+\nabla_{y} \gamma\right)\right]_{Y_{s}}
\end{gathered}
$$

Here angular brackets still denote velocity-space averaging, and the square brackets denote the extrinsic coordinate-space averaging : $[\psi]_{Y_{i}}=\frac{1}{|Y|} \int_{Y_{i}} \psi(\mathbf{y}) d \mathbf{y}$. The two averaging procedures are intimately mixed since the gas is not in continuum regime inside the pores, so there is an implicit need of a space region of size $|Y|$ in order to perform a velocity-space averaging in a short enough time interval.

\subsection{Discussion}

\subsubsection{Comparison with existing models}

The macroscopic relations yield a formal result that is similar to the approaches presented in the preceding sections. For instance, the extrinsic fluid velocity is :

$$
\mathbf{v}=-\underline{\underline{D}} \frac{\nabla P}{P}-\left(\underline{\underline{D}} \frac{T}{\rho}-\underline{\underline{D}}\right) \frac{\nabla T}{T}
$$

A term by term identification with the DGM relation (4) is then possible. Two remarks arise immediately. First, $\underline{\underline{D}}$ and $\underline{\underline{\underline{D}}}$ should be functions of pressure and temperature.

The precise dependence of the transport coefficients on $P$ and $T$ will be obtained numerically (Vignoles et al., 2007) ; however, the study of limiting regimes will help to check the validity of the model. This will be done in the following sections.

Second, the permeability, Knudsen diffusion coefficient, and thermal diffusion ratio may be extracted from $\underline{\underline{D}}$ and $\underline{\underline{D}}$. It is then possible to have more precise information about the tensorial character of these three quantities in a general porous medium. Examination of the closure problems (68) and (69) in the rarefied limit shows that these problems differ by the fact that, unlike $\beta$, the thermal transpiration variable $\alpha$ is couple to the solid-phase thermal conduction variable $\gamma$ 
through the interfacial boundary condition. Accordingly, the resulting fields may not be directly similar in a complex porous medium, and the tensors $\underline{\underline{D}}$ and $\underline{\underline{D}}$ may differ by their principal directions and anisotropy ratios. Again, this will be verified numerically in the companion paper (Vignoles et al., 2007).

\subsubsection{Rarefied limiting regime}

In the rarefied limit $(K n \rightarrow \infty)$, the closure problem (69) is simpler since the linearized operator $L(f)$ may be taken equal to zero, but one extra hypothesis of finite horizon has to be made in order to obtain a convergence of $D$ towards a finite value.

In a straight pore, an analytical formula is obtained for $\beta$. Its offaxis components are null, and the axial $(z)$ component obeys the following equation :

$$
\left\{\begin{array}{rlrl}
|\hat{\mathbf{c}}| \nabla_{\mathbf{n}(\hat{\mathbf{c}})} \beta_{z}= & -c_{z} & & \text { in } Y_{f} \\
\beta_{z}= & \sigma \int_{\mathbf{w} \cdot \mathbf{n}<0} \beta_{z}|\mathbf{w} \cdot \mathbf{n}| M_{n}\left(T_{0}\right) d \mathbf{w} & \\
& +(1-\sigma) \beta_{z}(\mathbf{y}, \mathbf{c}-2 \mathbf{c} \cdot \mathbf{n} \mathbf{n}) & & \text { in } \partial Y_{f s} \\
& & \text { and } \mathbf{c} \cdot \mathbf{n}>0
\end{array}\right.
$$

where $\hat{\mathbf{c}}$ stands for the vector $\left(\mathbf{c}_{x}, \mathbf{c}_{y}, 0\right)$. Let us define the intersection point of a particle lying at $\mathbf{y}$ with the wall $\zeta(\mathbf{y}, \mathbf{n c})$; a solution of (78) is :

$$
\beta(\mathbf{y}, \mathbf{c})=\frac{c_{z}}{|\hat{\mathbf{c}}|}|\mathbf{y}-\zeta|
$$

where it is seen that the half-chord length appears. Evaluation of the effective coefficient $\underline{\underline{D}}$ is then carried out :

$$
\begin{aligned}
D_{z z} & =\frac{1}{Y_{f}} \int_{Y_{f}} \int_{\mathbb{R}_{\mathbf{c}}^{3}} c_{z} \beta_{z} f_{0} d \mathbf{c} d \mathbf{y} \\
& =\sqrt{\frac{\mathcal{R} T_{0}}{8 \pi M}} \frac{1}{Y_{f}} \int_{Y_{f}} \int_{\omega=0}^{2 \pi}|\mathbf{y}-\zeta(\mathbf{y}, \mathbf{n}(\omega))| d \omega d \mathbf{y}
\end{aligned}
$$

The integral in (80) is, up to a proportionality factor, the chord length average of the pore. This result is identical to the result of Kennard (Kennard, 1938). In the case of a cylindrical pore, the final result is :

$$
D_{11}=\frac{1}{3} \bar{c} \cdot d_{p}
$$

which is identical to the Knudsen diffusion coefficient. 
The evaluation of coefficient $\tilde{D}_{11}$ is very similar to the preceding development, since eqs. (71) and (69) only display minute differences. Indeed, the modified variable $\alpha_{z}\left(-\frac{3}{2 T_{0}}+\frac{M|c|^{2}}{2 \mathcal{R} T_{0}^{2}}\right)^{-1}$ obeys strictly the same equation as $\beta_{z}$ in the fluid, and the boundary condition differs only by the addition of $\gamma_{z}$ to $\beta_{z}$ in the left-hand side of eq. (69). However, $\gamma_{z}$ is constant in a straight tube, and hence has no influence on the computation of $\tilde{D}$. Thus, in the rarefied limit, since $<|c|^{2}>$ tends to $2 \mathcal{R} T_{0} / M$, the ratio $\frac{\tilde{D}_{11} T_{0}}{D_{11} \rho_{0}}$ tends to $1 / 2$, as predicted by the DGM.

\subsubsection{Continuum limiting regime}

In the case of small Knudsen number (or small collision time $\tau$ ), the closure variables may be rescaled using $\tau$ as a small parameter. In the BGK case, by comparison of eqs. (71-70) and (66), it is recognized that (dropping out indices $i$ for sake of simplicity) :

$$
\begin{aligned}
\alpha & =\frac{\alpha^{(-1)}}{\tau}+\alpha^{(0)}+\tau \alpha^{(1)}+\ldots \\
\beta & =\beta^{(0)}+\tau \beta^{(1)}+\ldots \\
\gamma & =\gamma^{(0)}+\tau \gamma^{(1)}+\ldots
\end{aligned}
$$

with :

$$
\begin{aligned}
\alpha^{(-1)} & =b_{0}+\mathbf{b}_{1} \cdot \mathbf{c}+b_{2}|c|^{2} \\
\beta^{(0)} & =d_{0}+\mathbf{d}_{1} \cdot \mathbf{c}+b_{2}|c|^{2}
\end{aligned}
$$

and $\gamma^{(0)}$ is not coupled with $\alpha$. Moreover, from the boundary condition on $\alpha$ in eq. (71), it appears that $\left.\mathbf{b}_{1}\right|_{\partial Y_{f s}}=\mathbf{0}$ and $\left.b_{2}\right|_{\partial Y_{f s}}=$ 0 .

The linearized operator term in eq. (71) reads :

$$
L\left(\alpha f_{0}\right)=\frac{f_{0}}{\tau}(\frac{1}{\rho_{0}} \underbrace{\left(\begin{array}{c}
\frac{5}{2}-\frac{M|c|^{2}}{2 \mathcal{R} T_{0}} \\
\frac{M \mathbf{c}_{0}}{\mathcal{R} T_{0}} \\
\frac{M}{\mathcal{R} T_{0}}\left(\frac{M|c|^{2}}{3 \mathcal{R} T_{0}}-1\right)
\end{array}\right)}_{\rho_{0} \vec{d}} \cdot \underbrace{\left(\begin{array}{c}
\left\langle\alpha f_{0}\right\rangle \\
\left\langle\alpha f_{0} \mathbf{c}\right\rangle \\
\left\langle\alpha f_{0}|c|^{2} / 2\right\rangle
\end{array}\right)}_{\vec{\rho}\left[\alpha f_{0}\right]}-\alpha)
$$




$$
=\frac{f_{0}}{\tau}\left(\vec{d} \cdot \vec{\rho}\left[\alpha f_{0}\right]-\alpha\right)
$$

where some "arrow" notations have been introduced for sake of brevity.

At order -2 in $\tau, L\left(\alpha^{(-1)} f_{0}\right)=0$, and at order -1 in $\tau, L\left(\beta^{(0)} f_{0}\right)=$ 0 , from which one may deduce eqs. (85). Then, at order -1 , one has :

$$
\mathbf{c} \cdot \nabla_{y} \alpha^{(-1)}=\vec{d} \cdot \vec{\rho}\left[\alpha^{(0)} f_{0}\right]-\alpha^{(0)}
$$

The Fredholm alternative implies, since

$$
\left\langle\left(\vec{d} \cdot \vec{\rho}\left[\alpha^{(0)} f_{0}\right]-\alpha^{(0)}\right) f_{0} \vec{m}(\mathbf{c})\right\rangle=\overrightarrow{0}
$$

or equivalently

$$
\vec{\rho}\left[\vec{d} \cdot \vec{\rho}\left[\alpha^{(0)} f_{0}\right]-\alpha^{(0)}\right]=\overrightarrow{0},
$$

that the following condition be verified :

$$
\vec{\rho}\left[\mathbf{c} \cdot \nabla_{y} \alpha^{(-1)}\right]=\overrightarrow{0}
$$

Then, for some $e_{0}, \mathbf{e}_{1}$, and $e_{2}$ :

$$
\alpha^{(0)}=-\mathbf{c} \cdot \nabla_{y} \alpha^{(-1)}+e_{0}+\mathbf{e}_{1} \mathbf{c}+e_{2}|c|^{2}
$$

Recalling the structure at order -2 of $\alpha^{(-1)}$ (eq. (85)), relation (90) rewrites, after some algebra :

$$
\begin{aligned}
\nabla_{y} \cdot \mathbf{b}_{1} & =0 \text { in } Y_{f} \\
\mathbf{b}_{1} & =\mathbf{0} \text { on } \partial Y_{f s} \\
\nabla_{y}\left(b_{0}+\frac{5 \mathcal{R} T_{0}}{M} b_{2}\right) & =0 \text { in } Y_{f} \\
b_{2} & =0 \text { on } \partial Y_{f s}
\end{aligned}
$$

and eq. (92) is a continuity equation for $\mathbf{b}_{\mathbf{1}}$.

Now, at order 0 , and working the same way, one obtains :

$$
\mathbf{c} \cdot \nabla_{y} \alpha_{i}^{(0)}=c_{i}\left(\frac{M|c|^{2}}{2 \mathcal{R} T_{0}}-\frac{3 M}{2 \mathcal{R} T_{0}}\right)+\left(\vec{d} \cdot \vec{\rho}\left[\alpha^{(1)} f_{0}\right]-\alpha^{(1)}\right)
$$

This equation can be solved in $\alpha^{(1)}$ like eq. (89) has been solved in $\alpha^{(0)}, i$. e . using the Fredholm alternative again, and the result is :

$$
\vec{\rho}\left[\mathbf{c} \cdot \nabla_{y} \alpha_{i}^{(0)}-c_{i}\left(\frac{M|c|^{2}}{2 \mathcal{R} T_{0}}-\frac{3 M}{2 \mathcal{R} T_{0}}\right)\right]=\overrightarrow{0}
$$


Making use of eqs. (85) and (91), and restoring the use of indices $i$, one obtains :

$$
\begin{aligned}
-\nabla_{y}^{2} \mathbf{b}_{1 i}+\nabla_{y} \pi & =\frac{M}{\mathcal{R} T^{2}} \mathbf{x}_{i} \text { in } Y_{f} \\
\nabla_{y} \cdot \mathbf{b}_{1 i} & =0 \text { in } Y_{f} \\
\mathbf{b}_{1 i} & =\mathbf{0} \text { on } \partial Y_{f s}
\end{aligned}
$$

, where $\pi$ is a Lagrange multiplier which is a function of $e_{0}$ and $e_{2}$, and $\mathbf{x}_{i}$ is the $i^{\text {th }}$ vector of the canonical basis. Clearly, eqs. (98) are Stokes equations on $\mathbf{b}_{\mathbf{1}}$.

Coefficients $b_{0}$ and $b_{2}$ may also be computed, but they do not bring any contribution to $\underline{\underline{D}}$ since $f_{0}$ is an even function in c. After having dealt with the closure variable $\alpha$, the same overall procedure may be repeated for $\beta$; the difference being that there is no term at order -1 for it (see eq. (82)). This comes from the right-hand side of eq. (69) which contains a $1 / \rho_{0}$ additional term with respect to eq. (71), which can also be rewritten $(\mathcal{R} T / M \eta) \tau$. The result is that $\mathbf{d}_{1}$ in eq. (82) is solution of the same system as (98) but with a right-hand side in the first equation equal to $\eta^{-1} x_{i}$ instead of $\frac{M}{\mathcal{R} T^{2}} x_{i}$.

The final step of the procedure is now to compute the coefficients $\underline{\underline{D}}$ and $\underline{\underline{D}}$ using the first terms of the expansion for small $\tau$ :

$$
\begin{gathered}
\underline{\underline{D}}=\left\langle-f_{0} \mathbf{c} \otimes\left[\beta^{(0)}\right]_{Y_{f}}\right\rangle \\
\underline{\underline{\tilde{D}}}=\left\langle-f_{0} \mathbf{c} \otimes\left[\frac{\alpha^{(-1)}}{\tau}\right]_{Y_{f}}\right\rangle
\end{gathered}
$$

The relation between $\alpha^{(-1)}$ and $\beta^{(0)}$ implies that, for small $\tau$ :

$$
\underline{\underline{D}}=\frac{T_{0}}{\rho_{0}} \underline{\underline{D}}
$$

Accordingly, the velocity average of the fluid (eq. 72) may be rewritten

$$
\rho_{0} \mathbf{u}=-\underline{\underline{D}} \nabla_{x} \rho_{0}-\underline{\underline{D}} \nabla_{x} T_{0}=-\underline{\underline{D}} \rho_{0} \frac{\nabla_{x} P}{P}=-\rho_{0} \eta^{-1} \underline{\underline{B}} \nabla_{x} P
$$

which is indeed Darcy's law in which the permeability $\underline{\underline{B}}$ is obtained by averaging of the closure variables $\mathbf{b}_{1}$ :

$$
B_{i j}=\left[\left(\mathbf{d}_{1 i}\right)_{j}\right]_{Y_{f}}
$$


This ends the proof that the continuum regime limit of the presented model is coherent with past presentations of viscous transport descriptions. The same procedure may be carried out with the ES-BGK model, with identical results.

\section{Conclusion}

The non-isothermal transport of a pure rarefied gas in a porous medium has been treated with a change-of-scale procedure starting from the kinetic-theory level of description, and making use of the homogenization technique. Macroscopic equations and effective coefficients have been formally produced and compared to the popular "DustyGas Model" formalism. The relations found in this work are globally coherent with the DGM model, and the study of the model properties in the rarefied and continuum limiting cases gives the well-known laws for the fluxes (respectively Fick's law with a Knudsen diffusion coefficient on one hand and Darcy's law on the other).

The model has the potential to bring a more precise insight into the dependence of the coefficients to the geometry of a porous medium. In particular, it is seen that the thermal transpiration coefficient has indeed a tensorial nature ; in the trivial case of the rarefied limit in a straight cylindrical pore, the influence of the porous medium geometry is equivalent for Knudsen transport and for thermal transpiration is the same ; however, this is not true in general.

The presented approach has potential applications not only in the case of porous materials but also in micro-fluidics, $i$. e. gas flow in micro-domains (Haddad et al., 2005), providing that the flow has equivalent pore Knudsen number. Extensions to non-Darcian continuous behavior, $i$. e. Forchheimer correction, compressibility effects, etc ... are feasible along the same guidelines, by taking other scalings like higher Reynolds or Mach numbers and applying the same procedure.

The exploitation of this approach requires a numerical implementation of the closure problems : this will be presented in a companion paper (Vignoles et al., 2007), where the method is presented, validated and tested on some complex 3D images of porous media. 


\section{Acknowledgements}

The authors wish to thank CEA (Commissariat à l'Energie Atomique) for support through a PhD grant to C. P. and G. Duffa for fruitful discussions. 


\section{References}

Bardos, C., L. Dumas, and F. Golse: 1997, 'Diffusion approximation for billiards with totally accommodating scatterers'. J. Statis. Phys. 86, 351-375.

Bensoussan, A., J.-L. Lions, and G. C. Papanicolau: 1978, Asymptotic analysis for periodic structures, No. 5 in Studies in Mathematics and its Applications. Amsterdam: North-Holland.

Bruneton, E., B. Narcy, and A. Oberlin: 1997, 'Carbon-carbon composites prepared by a rapid densification process'. Carbon 35(10-11), 1593-1611.

Burganos, V. N. and S. V. Sotirchos: 1989, 'Knudsen diffusion in parallel, multidimensional, or randomly oriented pore structures'. Chem. Eng. Sci. 44, 2451.

Cercignani, C.: 1987, The Boltzmann Equation and its Applications, Vol. 67 of Applied Mathematical Sciences. Berlin: Springer Verlag.

Chapman, S. and T. Cowling: 1970, Mathematical thoery of nonuniform gases. Cambridge: Univ. Press, 3rd edition.

Charrier, P. and B. Dubroca: 2003a, 'Asymptotic transport models for heat and mass transfer in reactive porous media'. C. R. Acad. Sci. I 336, 537-542.

Charrier, P. and B. Dubroca: 2003b, 'Asymptotic transport models for heat and mass transfer in reactive porous media'. SIAM J. Multiscale Model. Simul. 2(1), 124-157.

Froment, G. F. and K. B. Bischoff: 1979, Chemical Reactor Analysis and Design. New York: John Wiley \& sons.

Golecki, I.: 1997, 'Recent advances in rapid vapor-phase densification of hightemperature fiber-matrix composites'. In: J. P. Singh (ed.): Proc. 21st Annual Conference and Exposition on Composites, Advanced Ceramics, Materials and Structures - B, Vol. 18 of Ceram. Eng. Sci. Proc. Westerville, OH: The American Ceramic Society, pp. 721-730.

Gupta, A. D. and T. S. Storvick: 1970, 'Analysis of the heat conductivity data for polar and nonpolar gases using thermal transpiration measurements'. J. Chem. Phys. $\mathbf{5 2}(2), 742-749$.

Haddad, O. M., M. M. Abuzaid, and M. A. Al-Nimr: 2005, 'Developing free convection gas flow in a vertical open-ended micro-channel filled with porous media'. Numerical heat Transfer A48(7), 693 - 710.

Haddad, O. M., M. A. Al-Nimr, and J. S. Al-Omary: 2007, 'Forced convection of gaseous slip-flow in porous micro-channels under Local Thermal Non-Equilibrium conditions'. Transport in Porous Media 67(3), $453-471$.

Hirschfelder, J. O., C. F. Curtiss, and R. B. Bird: 1963, Molecular theory of gases and liquids. John Wiley \& sons, $2^{\text {nd }}$ edition.

Kennard, E. H.: 1938, Kinetic Theory of Gases. New York: McGraw-Hill.

Kerkhof, P. J. A. M.: 1996, 'A modified Maxwell-Stefan model for transport through inert membranes: the Binary Friction Model'. The Chemical Engineering Journal 64, 319-343.

Kerkhof, P. J. A. M.: 1997, 'New light on some old problems : Revisiting the Stefan tube, Graham's law, and the Bosanquet equation'. Ind. Eng. Chem. Res. 36, 915-922.

Lackey, W. J.: 1989, 'Reviews, status, and future of the chemical vapor infiltration process for fabrication of fiber-reinforced ceramic composites'. Ceram. Eng. Sci. Proc. 10(7-8), 577-584.

Lamouroux, F. and G. Camus: 1994, 'Kinetics and mechanisms of oxidation of 2D woven C/SiC composites: . I. : Experimental approach.'. J. Amer. Ceram. Soc. 77, 20492057. 
Leutard, D., G. L. Vignoles, F. Lamouroux, and B. Bernard: 2002, 'Monitoring density and temperature in $C / C$ composites elaborated by CVI with radio-frequency heating'. J. Mater. Synth. and Proc. 9(5), 259-273.

Loyalka, S. K.: 1969, 'Thermal transpiration in a cylindrical tube'. Phys. Fluids 12, 2301.

Mason, E. A. and A. P. Malinauskas: 1983, Gas transport in porous media: the Dusty-Gas Model, Chemical Engineering Monographs. Elsevier.

Mason, E. A., A. P. Malinauskas, and R. B. E. III: 1967, 'Flow and diffusion of gases in porous media'. J. Chem. Phys. 46(8), 3199-3216.

Narcy, B., F. Guillet, F. Ravel, and P. David: 1995. In: R. R. Naslain and A. G. Evans (eds.): High-Temperature Ceramic Matrix Composites II, Vol. 58 of Ceram. Trans. Westerville, OH, USA, p. 237, The American Ceramic Society.

Naslain, R.: 1999, 'Processing of ceramic matrix composites'. In: Key Engineering Materials, Vol. 164-165 of Key Engineering Materials. Switzerland, pp. 3-8, Trans Tech Publications.

Naslain, R. and F. Langlais: 1990, 'Fundamental and practical aspects of the chemical vapor infiltration of porous substrates'. High Temperature Science 27, 221-235.

Reid, R. C., J. M. Prausnitz, and B. E. Poling: 1987, The properties of gases and liquids. McGraw Hill Book Company, 4th edition.

Sharipov, F.: 1996, 'Rarefied gas flow through a long tube at any temperature ratio'. J. Vac. Sci. Technol. A 14(4), 2627-2635.

Skjetne, E. and J.-L. Auriault: 1999, 'New insights on steady, nonlinear flow in porous media'. Eur. J. Mech. B : Fluids 18(1), 131-145.

Strieder, W. C.: 1971, 'Gaseous self-diffusion through a porous medium'. J. Chem. Phys. 54(9), 4050-4053.

Strieder, W. C. and S. Prager: 1968, 'Knudsen flow through a porous medium'. Phys. Fluids 11(12), 2544-2548.

Tomadakis, M. M. and T. J. Robertson: 2005, 'Viscous permeability of random fiber structures : comparison of electrical and diffusional estimates with experimental and analytical results'. J. Composite Mater. 39(2), 163-188.

Tomadakis, M. M. and S. V. Sotirchos: 1991, 'Effective Knudsen diffusivities in structures of randomly overlapping fibers'. AIChE J. 37, 74-86.

Vaidyaraman, S., W. J. Lackey, G. B. Freeman, P. K. Agrawal, and M. D. Langman: 1995, 'Fabrication of carbon-carbon composites by forced flow-thermal gradient chemical vapor infiltration'. J. Mater. Res. 10(6), 1469-1477.

Vignoles, G. L., P. Charrier, C. Preux, and B. Dubroca: 2007, 'Rarefied pure gas transport in non-isothermal porous media : Validation and tests of the model'. submitted to Transp. in Porous Media.

Whitaker, S.: 1987. In: S. Whitaker and A. Cassano (eds.): Chemical Reactor Analysis : Concept and Design, Vol. 3 of Chemical Engineering Concepts and Reviews. New York: Taylor \& Francis, Chapt. 1, pp. 1-94. 